\title{
Justicia social y gastronomía
}

El hoyo | Galder Gaztelu-Urrutia | 2019

\author{
Nadia McGowan
}

Universidad Internacional de la Rioja, España

Recibido: 8 de noviembre 2019; aceptado: 13 de enero 2020

\begin{abstract}
Resumen
The Platform/El hoyo es una fábula de terror que utiliza la comida como metáfora para criticar el sistema social actual y la falta de un reparto equitativo de recursos limitados. En ella, el monstruo deriva de las reacciones de los propios personajes al verse excluidos de los escalafones más ricos de la sociedad carcelaria que habitan. Ante esta situación, se plantean diferentes reacciones: la lucha individual por la supervivencia, las tentativas de concienciación social y el intento de revolución social. La historia da al espectador la oportunidad de realizar estas reflexiones morales utilizando el terror como plataforma para ello, pero sin llegar a una conclusión firme de qué camino se puede seguir para conseguir un futuro que se aleje de dicho género. En este artículo se analizan las implicaciones de las acciones de los diversos personajes, utilizando referentes literarios y sociales, poniendo especial énfasis en el uso de los alimentos por ser el eje principal que motiva y mueve a los personajes a la vez que crea dilemas éticos.
\end{abstract}

Palabras clave: El hoyo | comida | dilema moral | reparto de recursos

Social justice and gastronomy

\begin{abstract}
The Platform is a horror fable that uses food as a metaphor in order to criticize the current social system and its lack of an equitable distribution of limited resources. In this film, the monster arises from the reactions of its different characters when they find themselves excluded from the richest echelons of the prison they inhabit. Faced with this situation, several reactions arise: the individual struggle for survival, attempts towards creating social awareness and to start a social revolution. The story gives the viewer the chance to reflect on the moral implications of each part of the story, which uses horror to communicate its message while not reaching a clear conclusion as to which would be the best way to achieve a future different from that one portrayed. This article analyzes the implications of the actions of the different characters, using literary and social references, placing special emphasis on the use of food as the main element that motivates and moves the characters and creates ethical dilemmas.
\end{abstract}

Keywords: The Platform | food | moral dilemma | resource distribution

\section{Introducción}

The Platform/El hoyo (Gaztelu-Urrutia, 2019) narra una fábula en la que un grupo de prisioneros habitan celdas apiladas verticalmente en una torre de la que se desconoce cuántos niveles hay. Cada día un festín desciende desde el primer nivel sobre una plataforma y para unos minutos en cada uno para que las personas que hay en él puedan comer. Mientras los niveles superiores pueden alimentarse cómodamente, los inferiores se encuentran con el hambre. Los prisioneros permanecen en el nivel en que se encuentran durante un mes y, pasado éste, despiertan en otro aleatorio, con el subsiguiente cambio de fortuna. Encontrarse en un nivel inferior implica que la plataforma puede llegar sin alimento alguno y, por tanto, la muerte por inanición en un espacio vacío cuyo único cambio es la presencia (o no) de comida.

La película aparece clasificada en la IMDb como thriller y ciencia ficción. Kinglsey Amis definió la ciencia ficción en su obra New Gates of Hell de la siguiente manera:

La ciencia ficción es ese tipo de prosa narrativa que trata una situación que no podría darse en el mundo que conocemos pero sobre la cual se desarrolla una hipótesis basada en alguna innovación de la ciencia o tecnología, o pseudociencia o pseudotecnología, bien sea de origen humano o extraterrestre. ${ }^{1}$ (Amis, 2012)

Bajo esta definición no puede considerarse que esta obra sea ciencia ficción ya que su eje no son las conse-

*nadia.mcgowan@unir.net 
cuencias del desarrollo tecnológico y su única conexión con el género es su posible situación en un tiempo futuro, pero el cual sería irrelevante al argumento. La situación temporal puede ser irrelevante a la ciencia ficción, como demuestran series como Black Mirror (Brooker, 2011- ), que en algunos capítulos como The National Anthem (Bathurst, 2011) demuestran cómo ésta puede situarse en el presente.

El thriller, según Sánchez Noriega y Gubern (2012), se caracteriza por abordar "sucesos criminales o que entrañan amenazas de muerte" y se vertebra "a través de la personalidad singular de los protagonistas" (p. 159). Si bien existen elementos de suspense criminal en esta obra, es posible que su eje narrativo principal sea "provocar miedo e incomodidad en el espectador" y expresar "preocupaciones y miedos del presente, incluso en ocasiones hay relatos que constituyen auténticas alegorías de hechos históricos" (p. 153). En este contexto que se propone The Platform como una película de terror que utiliza la comida como elemento principal de una alegoría cuyo objetivo es realizar una profunda crítica social.

La película compitió en el festival de Sitges junto con Bacurau (Dornelles y Mendonça Filho, 2019) y Le Daim (Dupieux, 2019), entre otras. En estas dos obras se puede encontrar también crítica a distintos niveles del mundo que nos rodea. Bacurau presenta una crítica a la situación sociopolítica de Brasil en general y al gobierno de Jair Bolsonaro en particular, a través de la situación de un pueblo situado en el nordeste del país, su zona menos desarrollada. Le daim parte de la comedia para presentarnos a un hombre obsesionado con que su chaqueta de piel de ciervo sea admirada. A partir de esa premisa, la película desciende al horror ante los extremos a los que es capaz de llegar el personaje en una crítica a los valores actuales centrados en la apariencia.

Podemos encontrar numerosos ejemplos que aúnan cine y dilemas morales en tiempos recientes. Eye in the Sky (Hood, 2015) nos habla de los dilemas que la guerra y sus víctimas. Passengers (Tyldum, 2016) reflexiona sobre quitar la capacidad a otro de decidir su destino. Circle (Hann y Miscione, 2015) fuerza a quince personas a decidir quién será el próximo en morir y, con ello, reflexiona sobre qué valor damos a las personas y nuestros prejuicios.

Estas películas ilustran la preocupación planteada desde la Grecia antigua entre moral y razón práctica, como en La República (Platón, 2006). Las razones para actuar de forma moral suelen caen en dos categorías. En la primera, el acto inmoral no compensa por cómo nos afecta. En la segunda, el riesgo no compensa la posibilidad de ser descubierto. En el caso de The Platform se suma una variable y es la falta de consecuencias ante las acciones inmorales. Es por ese motivo que resulta relevante, en este momento, distinguir entre ética y moral.

Podría decirse que la ética tiene, con respecto a la moral, la misma relación que la filosofía de la ciencia con respecto a la ciencia (Uromson y Rée, 2005). Esta rama de la filosofía estudia la moral, entre otras cuestiones, sobre las cuales reflexiona. Los principios éticos serían universales y permanentes. La moral, en cambio, se refiere a los principios que guían el comportamiento de un grupo o individuo, con lo que la moral se refiere al comportamiento en un contexto social y temporal concreto.

En el APA Dictionary of Psychology se define "dilema ético" de la siguiente manera:

Una situación en la que dos principios morales entran en conflicto entre sí. Los dilemas ficticios o hipotéticos de este tipo se utilizan a menudo para evaluar las creencias morales o la capacidad de razonamiento moral de los individuos. También llamado dilema moral. ${ }^{2}$ (VandenBos y American Psychological Association, 2007)

El diccionario Oxford define "dilema moral" (no hay entrada para "dilema ético") de la siguiente manera: "Situaciones en las que cada posible línea de acción viola algún principio moral imperativo" ${ }^{3}$ (Blackburn, 2008). El diccionario de Blackwell (Bunnin y Yu, 2004) tampoco contempla "dilema moral" entre sus entradas. Cabe pensar, ante esto, que si bien existen diferencias entre ética y moral, puede haber superposición entre estos términos. La distinción se encontraría en si su esfera es la universal $\mathrm{o}$ la individual y social.

\section{El monstruo}

Ninguna película de terror está completa sin un monstruo que atente contra la integridad física, emocional o moral de los personajes. La tipología de éstos ha variado cuáles han sido los miedos generalizados en una sociedad según su momento histórico. Así podemos localizar el auge de películas de zombis en el presente siglo en relación con la crisis económica y como crítica al capitalismo que la provoca (Ferrero y Roas, 2011; Lauro y Embry, 2008).

En las primeras películas mudas el monstruo era un ser ajeno y exótico, otro, ejemplificado mediante el exótico Conde Drácula o el salvaje King Kong. Ante la amena- 
za comunista aparecen las películas como Invaders from Mars/ Invasores de Marte (Menzies, 1953), When Worlds Collidel Cuando los mundos chocan (Maté, 1951), Red Planet Mars (Horner, 1952), Them!/La bumanidad en peligro (Douglas, 1954), Earth vs. the Flying Saucers/La Tierra contra los platillos volantes (Sears, 1956) o Invasion of the Body Snatchers/La invasion de los ladrones de cuerpos (Siegel, 1956) donde los alienígenas destruyen los símbolos del modo de vida americano o la tierra y amenazan con asimilarlos en algunos casos. En la actualidad, el monstruo es el propio género humano y su comportamiento individual y grupal el que amenaza nuestra integridad. Un ejemplo temprano de monstruo interno sería Alien/Alien: el octavo pasajero (Scott, 1979), donde el temido xenomorfo se gesta en el cuerpo de los tripulantes de la nave para después devorarlos.

En The Platform, la amenaza es facilitada por el contexto pero es el comportamiento de los individuos participantes el que convierte el lugar en un entorno terrorífico cuando existe la potencialidad de evitarlo. El monstruo es interno, individual y, mediante la adición de personas en el entorno crea una sinergia que lo magnifica hasta crear una pesadilla. Es quizá una encarnación de la famosa frase de Plauto (1894) "Lobo es el hombre para el hombre" ${ }^{4}$ (p. 66).

El elemento que provoca el comportamiento monstruoso es la competición por una cantidad de comida limitada pero que podría ser suficiente si todos los habitantes de la sociedad de The Platform tomaran sólo su parte. Esto provoca, a nivel individual, una duda moral similar a la del dilema del prisionero (Poundstone, 1992) y es su resolución y las posteriores reacciones las que, según su cariz moral, les convierten en seres monstruosos.

La investigación en torno al dilema del prisionero puede trazarse a los años 50 (Nash, 1959; Scodel et al., 1959). La investigación más reciente también ha indagado en qué ocurre cuando hay más de dos opciones y personas involucradas. Hay un área de estudio llamada dilema del prisionero iterativo (Axelrod, 1987) donde el dilema se plantea de forma repetida a los mismos participantes, lo cual permite que los participantes aprendan del comportamiento de los demás. Algunos estudios (Darwen y Yao, 2001) apuntan a que una mayor cantidad de opciones disminuye la cooperación. La cooperación parece depender de factores como la popularidad (Milgrom y Roberts, 1982), información incompleta (Harsanyi, 1967) o la racionalización limitada (Simon, 1955; Vegaredondo, 1994). Axelrod (1984) atribuye la cooperación a la "sombra del futuro", es decir, la probabilidad e importancia de interacciones futuras. Esto implica que el comportamiento dependerá, en gran medida, del interés personal y será una inversión de la que se espera rédito.

En el caso de esta película, encontramos este dilema con variaciones. No hay solo dos actores, sino múltiples. En cada nivel hay dos personas, con lo que una siempre acompaña al protagonista y crea una respuesta posible adicional. En lugar de limitarse a dos individuos racionales que se beneficiarían de la cooperación mutua, nos encontramos con una multitud cuya colaboración sería necesaria para el beneficio de todos.

La película comienza en la cocina de un restaurante de lujo, la cual se retrata con estilo publicitario mediante planos breves, muy delimitados, y con una fotografía más amable y cálida que el resto de ambientes del film. Una serie de cocineros preparan delicados platos, de aspecto decadente y opulento. Entre ellos, uno es amonestado por el jefe de cocina al haber dejado que un pelo le cayera en ella.

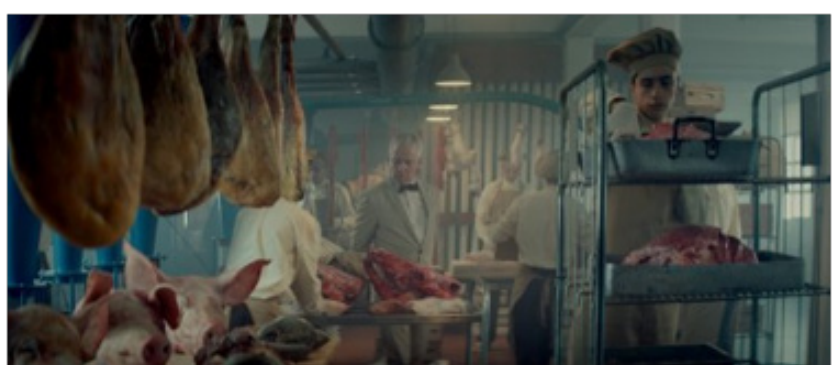

Figura 1: Restaurante opulento al inicio de la película

El espectador no es consciente en ese momento de la finalidad del banquete. Éste será transportado a lo largo de todas las celdas de la cárcel vertical, empezando por la más alta. Cada habitante tiene libertad de tomar lo que desee de cuanto le llegue, pero no puede quedarse con nada para después. Así pues, los que se sitúan en capas inferiores están a merced de las acciones de los niveles más altos y la comida funciona como un símbolo de exclusión y separación.

Esta metáfora podría estar representando el "efecto derrame”, según la cual la prosperidad de los más ricos gotea hasta los menos afortunados y les ayuda a todos. Esto es aplicado en la economía contemporánea mediante la reducción de impuestos a los sectores más ricos y a las grandes corporaciones bajo el argumento de fomentar la economía. Puede encontrarse ejemplo de ello en el trabajo de Arthur Laffer, impulsor de la implantación de medidas económicas en este sentido durante la administración de Reagan en Estados Unidos y consejero de economía del actual presidente Donald Trump (Moore y 
Laffer, 2018). Esta postura ha sido abundantemente criticada (Hirsch, 1980; Arndt, 1983; Dabla-Norris et al., 2015) pero, en la película, sólo cuestiona cómo los reclusos se relacionan entre sí. No se plantea si los recursos iniciales son suficientes, si debieran ser mayores o cómo pedirlos, sino cómo los diferentes niveles luchan entre sí por ellos. En ese sentido, no cuestiona al sistema sino tan sólo a las clases más pobres de éste y los conflictos entre ellas y los sitúa en un mundo globalizado como lo describía Zygmunt Bauman (2001):

Vivimos en un mundo globalizado. Esto significa que todos, seamos conscientes de ello o no, dependemos los unos de los otros. Cualquier cosa que hagamos o nos abstengamos de hacer afecta la vida de personas que viven en lugares que nunca visitaremos. Y lo que sea que hagan estas personas distantes o dejan de hacer, tendrá un impacto en las condiciones en las que cada uno de nosotros, juntos y por separado, conduzcamos nuestras vidas (...) Vivir en un mundo globalizado significa ser consciente del dolor, la miseria y el sufrimiento de innumerables individuos que nunca conoceremos en persona. ${ }^{5}$ (p. 16)

Los reclusos son, necesariamente, consumidores que, por supervivencia, deben participar en el sistema ya que excluirse de él implica la muerte por inanición. La comida contiene en sí el potencial de supervivencia, de conseguir al menos los objetivos de la base de la pirámide de Maslow (1943) que satisfacen las necesidades fisiológicas. A su vez, se presenta como un símbolo de todo lo deseado y deseable pero, a la vez, inalcanzable. Los personajes hacen referencias a lo que deben de percibir los que habitan los niveles superiores, pero jamás lo ven. Sí saben cuánto quedará para los siguientes y el sufrimiento de los que están por debajo de ellos, pero no siempre motiva una respuesta ética por su parte.

En un mundo de consumidores, los bienes y recursos limitados son el mayor deseo, cuya obtención trae la satisfacción inmediata. Antes de aceptar entrar al Hoyo, en la entrevista de acceso, se les pregunta también si tienen alergias, intolerancias y cuál es su plato preferido. Esto promete que los bienes estarán adaptados a la persona, diseñados para él. Similar a las promesas publicitarias de productos caros, no cuenta el coste personal de adquirirlas. En el caso de esta obra, obtenerlos es mera cuestión de suerte ya que no existen mecanismos que permitan acceder a ellos. Puede ser una crítica de un sistema desigual, en el que si bien se dice que el esfuerzo y el trabajo duro se verán recompensados, la realidad es muy distante, tal y como refleja la sociedad millennial, una generación con alta formación académica pero, económicamente, muy precaria (Burstein, 2013).

\section{Trimagasi}

La historia comienza con dos personajes en una celda, Goreng y Trimagasi. Este primer compañero de celda responde a las primeras iteraciones de un dilema del prisionero repetido a lo largo del a película.

El primer mes, su relación es afable conforme se alimentan de forma suficiente en un nivel abundante. En este caso, dispuestos ante una situación ventajosa, no cooperan con los menos afortunados aunque esto beneficiaría al conjunto. En el segundo mes, en cambio, despiertan en un nivel poco afortunado y experimentan la otra cara de la moneda.

Ante esta circunstancia, Trimagasi maniata a Goreng con la intención de cortar trozos de su carne para poder alimentarse. Podría relacionarse con el episodio retratado en el lienzo de Géricault titulado La balsa de la Medusa (1819) y el episodio real en que se inspiraba. Este cuadro muestra la balsa improvisada por las 147 personas que quedaron a la deriva tras el naufragio de la fragata Méduse, de las cuales murieron todas excepto 15 en las dos semanas que tardaron en rescatarlos. Trimagasi encarna el afán de supervivencia más primario, donde no hay cabida para moralidad ni ética alguna y, además, tampoco hay empatía hacia los que están en su misma situación.

Esto se ve reflejado en cómo trata el personaje la comida: la manipula con desprecio, escupe sobre los restos e insulta a los siguientes cuando la plataforma baja. Justifica esto aduciendo que "los de arriba" han hecho lo mismo antes que él, con lo que él participa de la cadena en la que el más fuerte oprime al que es más débil.

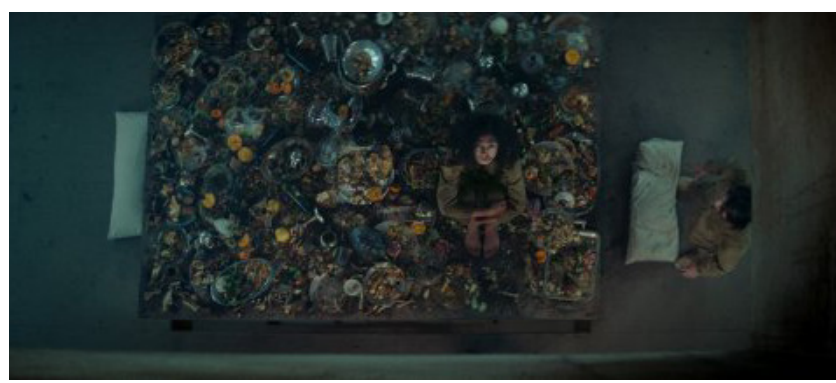

Figura 2: La plataforma de comida

Ante esta situación, la propia persona se puede tornar objeto. Kant (1996) consideraba que las cosas tenían precio, pero las personas tenían valor, ya que no son medios sino fines en sí mismas. Sin embargo, este entorno convierte al consumidor en objeto de consumo, en alimento, desproveyéndolo de toda dignidad. La reflexión que plantea esta secuencia es amplia por la 
casuística que genera, de nuevo derivada del dilema del prisionero. La propuesta que se realiza es, no obstante, cruel: Trimagasi planea cortar trozos del cuerpo de Goreng y alimentarlos a ambos de ellos. No se desvela si autofagocitarse hubiera permitido sobrevivir, porque el protagonista es rescatado de esta situación por Miharu, una mujer que busca a su hijo. Una vez liberado, Goreng acuchilla sin piedad a su agresor, en un arranque de violencia inesperado, que puede hacer pensar que si se utiliza a las personas como cosas, éstas no responderán de otra forma.

\section{Imoguiri}

Tras plantear a dónde lleva el afán por la mera supervivencia, la película introduce un nuevo personaje, llamado Imoguiri, quien intenta plantear un sistema de reparto justo. Esta iteración del dilema plantea la cooperación y los problemas que puede plantear en un sistema con múltiples actores. Este personaje proviene del interior del sistema que crea la sociedad desigual y, se presupone, participa de su razonamientos internos. Sin embargo, se muestra que desconoce el alcance de sus implicaciones, las cuales no ha podido apreciar hasta participar en el sistema.

Parfit analiza en su trabajo Reasons and Persons (2007) la resolución práctica de dilemas, especialmente con respecto a la contribución personal y es relevante en esta parte de la historia. Dice que "A menudo se afirma que, en aquellos dilemas en que participan muchas personas, lo que cada una haga no cambiará nada" ${ }^{6}$ (Parfit, 1984). Su texto continúa argumentando por qué esta idea es errónea. Una de las razones relevantes para este contexto es la que explica que:

Aunque una acción no perjudique a nadie, esta acción puede ser incorrecta porque es parte de un grupo de acciones que en conjunto [sic] perjudican a otras personas. Del mismo modo, incluso si un acto no beneficia a nadie, puede ser lo que uno debería hacer porque es parte de un conjunto de acciones que en conjunto benefician a otras personas. $^{7}$ (p. 70)

Imoguiri plantea dos estrategias de acción diferentes que parecen partir de este planteamiento. La primera de ellas es la acción individual e intentar concienciar al resto de habitantes. Hace eso preparando dos raciones de comida para los inquilinos del siguiente nivel y consumiendo sólo lo que necesita. Acto seguido, les explica lo que ha hecho y les pide que hagan lo mismo, con un lenguaje respetuoso y cuidado, totalmente descontextualizado para el lugar. La recepción no es positiva y sus esfuerzos son ignorados de forma repetida.

Esta recepción negativa podría situarse en otro error planteado por Parfit (1984), que dice que, en dilemas que involucran a mucha gente "Se afirma que, en estos casos, lo que cada persona hace no cambia nada" (p. 73) porque la probabilidad que tienen que causar un cambio es muy pequeña. Los prisioneros, en ese momento, no consideran que sus acciones puedan traer cambios a gran escala.

Argumenta Parfit que hay ocasiones en que, por pequeña que sea la probabilidad de causar un efecto, no se puede pasar por alto por lo crítico que sería el efecto causado. El ejemplo que elige es el de un ingeniero nuclear que tiene una posibilidad entre un millón de causar una catástrofe. En un caso así, no puede ignorarse una posibilidad, por pequeña que sea. Extiende este razonamiento a los casos que afectan a una gran cantidad de gente o que se extienden en el tiempo, por cómo se acumularían los beneficios.

La segunda acción es la amenaza de violencia. Este comportamiento no parece tener en cuenta las razones antes mencionadas para cooperar ante una situación de dilema de prisionero. El cambio de actitud se da cuando interviene Goreng, quien amenaza a los del nivel inferior con defecar sobre la comida hasta que atiendan a sus razones. Sin embargo, esto sólo garantiza el cambio en el nivel inmediatamente inferior y no hay garantías posteriores.

Suele asumirse que la violencia es causada por la deshumanización del otro, lo cual reduce la percepción de que se les deba un trato ético. Existen estudios (Rai, Valdesolo y Graham, 2017) que, en cambio, argumentan que la violencia puede tener motivaciones morales que lleven a ella. En este caso, los personajes no se benefician de forma material por sus acciones, como hubiera hecho el personaje caníbal, sino que recurren a las amenazas porque consideran que los otros la merecen, con lo que pueden considerarse virtuosos (Fiske y Ray, 2014).

\section{Baharat}

Baharat aparece escalando el agujero del hoyo con una cuerda como única posesión, en clara alusión al ascenso social. Ante sus intentos, los habitantes del piso superior fingen conversar entre sí, sopesando si ayudarle o no, hasta acceder a ello. Cuando está ascendiendo, le empujan de vuelta hacia abajo, entre risas y lanzándole heces. Ante la imposibilidad de conseguir colaboración superior, sólo queda como opción tomar la justicia por la propia mano. 
Es en ese momento que los personajes deciden tomar las riendas del reparto de comida y redistribuir ellos mismos los recursos. Parecería que la solución al dilema dada en la película es eliminar la libre elección para evitar que ésta no sea la cooperación. Narrativamente, parece más orientado a cambiar la historia hacia un viaje del héroe (Campbe11, 2003), especialmente notorio por la adquisición de un símbolo, un acompañante y una misión.

Deciden no repartir comida en los primeros cincuenta pisos, para compensar las injusticias pasadas. Esta reflexión plantea al espectador si pudiera ser justo causar hambre hoy a unos pocos por las inequidades pasadas provocadas por el sistema. En segundo lugar, aparecen personajes que han ayudado a Baharat en su ascenso, quienes apelan a su relación personal y ayuda previa. En la película, tampoco se les concede un trato de favor. Por último, aparece la figura del hombre sabio, quien les dice que necesitan un símbolo inmaculado, un plato que llegue sin haber sido tocado de vuelta, para que "los de arriba” sepan de su misión. Una panacota es el plato elegido, tratado como el Santo Grial.

La comida se ha utilizado como símbolo de protesta en tiempos recientes, como en la Fishball Revolution (Revolución de las bolas de pescado), situada en Hong Kong en febrero del 2016 o en el movimiento \#jedzjabłka del 2014. En ese año, Putin había prohibido la importación de frutas y verduras polacas, ante lo cual los polacos respondieron con tweets y posts comiendo manzanas, "jedzjabłka”. También puede aparecer como arma, como en el caso del milkshaking. Esta práctica consiste en lanzar un batido a modo de protesta, una práctica popularizada en el Reino Unido durante las elecciones parlamentarias de 2019 (Weaver, 2019) como forma de causar humillación pública (Chakelian, 2019). De modo similar, se han utilizado bombas de harina (Tempest, 2004) o huevos como armas arrojadizas (Fawkes, 2004). Las protestas causadas por el hambre han sido, históricamente, una fuente de revueltas, como las del 2011 en la plaza Tahrir de Egipto (Lagi, Bertrand y Bar-Yam, 2011). En Grecia, en el 2011, "comer" se usó como metáfora del consumo desmedido y, durante la crisis de deuda soberana la pregunta era quién se había comido el dinero (Sutton, 2011; Sutton et. al., 2013).

En este caso, la simbología muestra más relación con

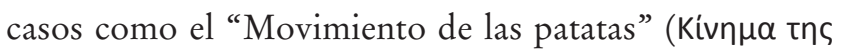

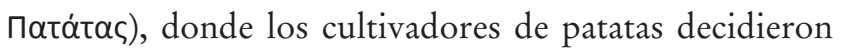
distribuirlas de forma gratuita para quejarse contra el gobierno y su situación económica (Henley, 2012) o las protestas de agricultores españoles (ABC, 2019). Sin embargo, no agotan la vía pacífica puesto que, para que el mensaje llegue intacto, los personajes luchan contra el resto de prisioneros.

Tras descender cientos de niveles, no queda nadie con vida, y la plataforma no se detiene hasta que llega al último. No parece haber nadie con vida pero, bajo la cama, hay un niño con vida. Esta situación plantea qué es más importante, si alimentar a la criatura o mantener el símbolo intacto. La decisión es pragmática: el niño está presente y es inmediato. Por ese motivo, se sacrifica el emblema de la pequeña revolución planteada para poder apenas saciarlo. Esto les deja desprovistos de cualquier avance y en la misma situación en la que estaban al comenzar. La película no plantea respuestas al reparto injusto de recursos, ni guías de comportamiento. El final es tremendamente abierto y sólo parece apuntar a que la próxima generación deberá enfrentarse al mismo reto, ya que la presente no ha sabido superarlo.

\section{Conclusiones}

La comida es parte intrínseca de la economía moral la sociedad y su reparto es indicativo de las relaciones que se establecen en su interior. The Platform plantea una distopía fantástica donde la comida es utilizada como elemento vertebrador y simbólico del consumo de recursos, cuya carencia hace que el espectador deba replantearse la racionalidad del mercado. El uso de la comida como elemento de exclusión llevará a su uso como símbolo de resistencia, en un intento de revolución entre los presos. Pueden encontrarse antecedentes recientes de usos simbólicos de la comida como elemento de protesta en la Fishball Revolution o el milkshaking, entre otros.

Su reparto sitúa al protagonista y a todos los participantes en el sistema en un dilema del prisionero iterativo y con un gran número de participantes. No sólo deben elegir si compartir justamente la comida y cooperar o no, sino que deben hacerlo múltiples veces, con el recuerdo de lo que el resto de participantes han hecho previamente. Esto lleva a la narración de diversas estrategias: la no cooperación egoísta, intentos de razonar por qué todos deben cooperar y, finalmente, la decisión del protagonista de usar la fuerza para forzar la cooperación por métodos violentos.

La película plantea varias críticas. La primera es la del "efecto derrame", según el cual la riqueza "gotea" hasta los más pobres, al mostrar su sufrimiento cuando no se colabora en un reparto justo. La segunda es la objetivación de las personas en dicha situación puesto que convierte a la persona en objeto de consumo, equiparándola 
a la comida. La tercera crítica es hacia los que opinan que un pequeño esfuerzo individual no puede cambiar nada, aunque esto termina respaldándose por la violencia. Finalmente, la solución por la que opta el protagonista es no permitir que el resto de participantes elijan qué curso de acción quieren tomar, con la intención de destruir el sistema en el que participan.
La película plantea, desde la crueldad del cine de terror, la lucha por la supervivencia, el intento de concienciación social y parece mostrar que la violencia es la única solución en situaciones cercanas al dilema del prisionero. Sin embargo, ésta también es fallida y nos deja sin más respuesta que el esperar que la próxima generación pueda hacerlo mejor que nosotros.

\section{Referencias}

$\operatorname{ABC}(2019,14$ de febrero). AVA reparte cuatro toneladas de naranja en Valencia como protesta a la gestión de la crisis agrícola. $A B C$. Recuperado de https:/Www.abc.es/espana/comunidad-valenciana/abci-reparte-cuatro-toneladas-naranja-valencia-como-protesta-gestion-crisis-agricola-201902141554_noticia.html

Alperson, E.L. (productor) y Menzies, W. (director). (1953). Invaders from Mars [cinta cinematográfica].Estados Unidos: 20th Century Fox.

Amis, K. (2012). New maps of hell: A survey of science fiction. Londres: Penguin books.

Arndt, H. W. (1983). The "trickle-down" myth. Economic Development and Cultural Change, 32(1), 1-10.

Axelrod, R. (1984). The Evolution of Cooperation. Nueva York: Basic Books.

Axelrod, R. (1987). The evolution of strategies in the iterated prisoner's dilemma. The dynamics of norms, 1-16.

Bauman, Z. (2001). The Moral Universe. Londres: Demos. Recuperado de: http://www.demos.co.uk/files/theMoraluniverse.pdf Blackburn, S. (2008). The Oxford Dictionary of Philosophy. Oxford: Oxford University Press.

Brooker, C. (creador). (2011- ). Black Mirror [serie televisiva]. Reino Unido: Channel 4; Netflix.

Bunnin, N. y Yu, J. (2004). The Blackwell Dictionary of Western Philosophy. Oxford: Blackwell.

Bursch, J., Einbinder, S., Nardelli, M., Nardelli, T., Stiefel, B. (productores) y Hann, A., Miscione, M. (directores). (2015). Circle [cinta cinematográfica]. Estados Unidos: FilmBuff.

Burstein, D. D. (2013). Fast Future: How the Millennial Generation is Shaping our World. Boston: Beacon Press.

Carroll, G., Giler, D., Hill, W. (productores) y Scott, R. (director). Alien [cinta cinematográfica]. Estados Unidos: 20th Century Fox. Chakelian, A. (2019, 16 de mayo). “Lactose against Intolerance!” How milkshakes became a tool of protest. NewStatesman. Recuperado de: https://www.newstatesman.com/politics/uk/2019/05/lactose-against-intolerance-how-milkshake-became-tool-protest

Campbell, B. (2003). The hero with a thousand faces. California: New World Library.

Dabla-Norris, M. E., Kochhar, M. K., Suphaphiphat, M. N., Ricka, M. F., y Tsounta, E. (2015). Causes and consequences of income inequality: A global perspective. International Monetary Fund. doi: 10.5089/9781513555188.006

Darwen, P., y Yao, X. (2001). Why More Choices Cause Less Cooperation in Iterated Prisoner's Dilemma. Proceeding of the 2001 Congress of Evolutionary Computation (987-994). Nueva Jersey: IEE Sercive Center.

Doherty, G., Firth, C., Lancaster, D. (productores) y Hood, G. (director). (2015). Eye in the sky [cinta cinematográfica]. Reino Unido: Enterteinment One; Raindog Films.

Fawkes, H. (2004, 24 de septiembre). Ukraine PM hurt in 'egg attack'. BBC News. Recuperado de: http://news.bbc.co.uk/2/hi/ europe/3686368.stm

Ferrero, Á., y Roas, S. (2011). El 'zombi' como metáfora (contra) cultural. Nómadas. Critical Journal of Social and Juridical Sciences, 32(4). doi: 10.5209/rev_NOMA.2011.v32.n4.38076

Fiske, A.P., y Rai, T.S. (2014). Virtuous Violence: Hurting and Killing to Create, Sustain, End, and Honor Social Relationships. Cambridge: Cambridge Univ Press.

Hamel, S., Maher, M., Marmur, O., Moritz, N. (productores) y Tyldum, M. (director). (2016). Passengers [cinta cinematográfica]. Estados Unidos: Columbia Pictures.

Harsanyi J. (1967) Games with incomplete information played by Bayesian players. Management Science, 14(3), $159-182$.

Henley, J. (2012, 27 de marzo). Greece's cut-price potato movement shows Greeks chipping in. The Guardian. Recuperado de https:// www.theguardian.com/world/2012/mar/27/greece-breadline-potato-movement-eurozone-crisis 
Hernández, A.; Juárez, C.; Matamoros, D. (productores) y Gaztelu-Urrutia, G. (director). (2019). The Platform [cinta cinematográfica]. Bilbao: Basque Films.

Hirsch, B. T. (1980). Poverty and economic growth: has trickle down petered out?. Economic Inquiry, 18(1), 151.

Hyde, D., Veiller, A. (productores) y Homer, H. (director). (1952). Red Planet Mars [cinta cinematográfica]. Estados Unidos: United Artists.

Kant, I. (1996). Fundamentos de la metafísica de las costumbres. Barcelona: Ariel.

Lagi, M., Bertrand, K. Z., y Bar-Yam, Y. (2011). The food crises and political instability in North Africa and the Middle East. Available at SSRN 1910031. doi: 10.2139/ssrn.1910031

Lauro, S. J., y Embry, K. (2008). A zombie manifesto: The nonhuman condition in the era of advanced capitalism. Boundary 2, 35(1), 85-108. doi: 10.1215/01903659-2007-027

Maslow, A.H. (1943). A Theory of Human Motivation. Psychological Review, 50(4), 370-396. doi: 10.1037/h0054346.

Mendonça Filho, K., y Dornelles, J. (directores). (2019). Bacurau [cinta cinematográfica]. Brasil: CinemaScópio Produções / SBS Films / Símio Filmes / arte France Cinéma.

Milgrom, P., y Roberts, J. (1982). Predation, reputation and entry deterrence. Journal of Economic Theory, 27, 280-312.

Moore, S., y Laffer, A. B. (2018). Trumponomics: Inside the America first plan to revive our economy. Nueva York: All Points Books. Nash, J. (1959). The Bargaining Problem. Econometrics, 18, 150-155.

Pal, G. (productor) y Maté, R. (director). (1951). When Worlds collide [cinta cinematográfica]. Estados Unidos: Paramount Pictures.

Parfit, D. (1984). Reasons and Persons. Oxford: Clarendon Press.

Platón (2006). La república. Pozuelo de Alarcón: Espasa.

Plauto, T.M. (1894). T. Macci Plavti Asinaria. Londres: Cambridge University Press.

Poundstone, W. (1992). Prisoner's Dilemma. Nueva York: Doubleday.

Rai, T. S., Valdesolo, P., y Graham, J. (2017). Dehumanization increases instrumental violence, but not moral violence. Proceedings of the National Academy of Sciences of the United States of America, 114(32), 8511-8516. doi: 10.1073/pnas.1705238114

Sánchez-Noriega, J. L., y Gubern, R. (2012). Historia del cine: Teoría y géneros cinematográficos, fotografía y televisión. Madrid: Alianza.

Schneer, C.H., Katzman, S. (productores) y Sears, F. (director). (1956). Earth vs. Flying Saucers [cinta cinematográfica]. Estados Unidos: Columbia Pictures.

Scodel, A.; Minas, J.S.; Ratoosh, P.; Lipetz, M. (1959). Some Descriptive Aspects of Two-Person, Non-Zero-Sum Games. Journal of Conflict Resolution, 3, 114-119.

Simon H. (1955). A behavioral model of rational choice. Quarterly Journal of Econometrics, 69(1), 99-118.

Sutton, D. (2011). Eating in Times of Financial Crisis [Blogpost]. Recuperado de: https://foodanthro.com/2011/06/27/eating-in-times-of-financial-crisis/

Sutton, D., Naguib, N., Vournelis, L., y Dickinson, M. (2013). Food and Contemporary Protest Movements. Food, Culture E Society, 16(3), 345-366. doi: 10.2752/175174413X13673466711642

Tempest, M. (2004, 20 de mayo). Two charged over flour bomb attack. The Guardian.

Uromson, J.O.; Rée, J. (2005). The Concise Enciclopedia of Western Philosophy and Philosophers. Londres, Nueva York: Routledge. VandenBos, G. R., y American Psychological Association. (2007). APA dictionary of psychology. Washington, DC: American Psychological Association.

Vegaredondo F. (1994). Bayesian boundedly rational agents play the infinitely repeated prisoner's dilemma. Theory and Decision 36(2), 187-206.

Verhaeghe, T. y Verhaeghe, M. (productores) y Dupieux, Q. (director). (2019). Le Daim [cinta cinematográfica]. Francia: Atelier de production.

Wanger, W. (productor) y Siegel, D. (director). (1956). Invasion of the Body Snatchers [cinta cinematográfica]. Estados Unidos: Walter Wanger Productions.

Weaver, M. (2019, 20 de mayo). Nigel Farage doused with milkshake in Newcastle. The Guardian. Recuperado de: https://www. theguardian.com/politics/2019/may/20/nigel-farage-doused-with-milkshake-in-newcastle

Weisbart, D. (productor) y Douglas, G. (director). (1954). Them! [cinta cinematográfica]. Estados Unidos: Warner Bros. 
Science fiction is that class of prose narrative treating of a situation that could not arise in the world we know, but which is hypothesized on the basis of some innovation in science or technology, or pseudo-science or pseudo-technology, whether human or extra-terrestrial in origin.

2 A situation in which two moral principles conflict with one another. fictional or hypothetical dilemmas of this kind are often used to assess the moral beliefs or moral reasoning skills of individuals. Also called moral dilemma.

3 Situations in which each possible course of action breaches some otherwise binding moral principle.

$4 \quad$ lupus est homo homini.

5 We live in a globalising world. That means that all of us, consciously or not, depend on each other. Whatever we do or refrain from doing affects the lives of people who live in places we'll never visit. And whatever those distant people do or desist from doing has its impact on the conditions in which we, each one of us separately and together, conduct our lives. Living in a globalising world means being aware of the pain, misery and suffering of countless people whom we will never meet in person.

6 it is often claimed that, in those contributor's dilemmas that involve very many people, what each person does would make no difference.

7 Even if an act harms no one, this act may be wrong because it is one of a set of acts that together [sic] harm other people. Similarly, even if some act benefits no one, it can be what someone ought to do, because it is one of a set of acts that together benefit other people.

$8 \quad$ it is often claimed that, in these cases, what each person does makes no diference. 